\title{
$\mathrm{N}$-linked glycosylation of proteins in the protozoan parasite
}

\section{Toxoplasma gondii}

\author{
Flora C. Y. Luk, Terezina M. Johnson, and Con J. Beckers ${ }^{1}$ \\ Department of Cell \& Developmental Biology, University of North Carolina, Chapel Hill, NC \\ 27599-7090, USA.
}

\section{Abstract}

Toxoplasma gondii is an obligate intracellular parasite of animal cells. Infection of humans is common and may result in devastating disease, especially in immunocompromised individuals. Despite previous reports that $\mathrm{N}$-glycosylation of proteins may be a rare post-translational modification in this and related organisms, we demonstrate that it is actually quite prevalent in Toxoplasma. N-glycosylation is completely inhibited by treatment of parasites with tunicamycin, but this does not appear to exert its major effect on the parasites until they have egressed from their host cells. Although the tunicamycin-treated parasites appear structurally normal at this time they are not motile and mostly incapable of invading new host cells. The few tunicamycin-treated parasites that do invade are severely affected in their ability to replicate and accumulate with a distended endoplasmic reticulum, deformed nuclei, and without recognizable late secretory organelles. We provide experimental evidence that indicate that Toxoplasma $\mathrm{N}$-glycans differ structurally from those in other eukaryotes.

\section{Introduction}

The protozoan parasite Toxoplasma gondii is an obligate intracellular parasite of humans and other animals. Infection with this parasite is common and can result in serious disease, although this is most likely to occur in individuals with a deficient immune system or in first-trimester fetuses [1,2]. Infection is typically initiated by the ingestion of food contaminated with either Toxoplasma oocysts or tissue cysts, containing sporozoites and bradyzoites respectively. Once inside the host, these differentiate into the rapid replicating tachyzoites that spread throughout the infected host [1,2]. Unlike other intracellular parasites that gain access to host cells through co-opting their endocytic or phagocytic apparatus, Toxoplasma and other apicomplexan parasites actively penetrate their host cells. In this process, they attach to the plasma membrane of host cells, and subsequently penetrate them while creating a vacuole, the parasitophorous vacuole, that they reside in throughout their stay inside the host cell [3].

All of these steps are intimately coupled to the secretion of material from the parasite. Toxoplasma tachyzoites contain an extensive secretory system consisting of an extensive endoplasmic reticulum, a Golgi apparatus, and multiple specialized late secretory organelles: the micronemes, dense granules, and rhoptries. Microneme proteins are required for parasite binding to host cells [4] but the exact function of the numerous rhoptry and dense granule proteins is unknown. Most rhoptry proteins are inserted into the PVM [5] but some are injected

\footnotetext{
${ }^{1}$ Corresponding author E-mail: cbeckers@med.unc.edu Phone: + 1919 966-1464 Fax: + 1919 966-1856

Publisher's Disclaimer: This is a PDF file of an unedited manuscript that has been accepted for publication. As a service to our customers we are providing this early version of the manuscript. The manuscript will undergo copyediting, typesetting, and review of the resulting proof before it is published in its final citable form. Please note that during the production process errors may be discovered which could affect the content, and all legal disclaimers that apply to the journal pertain.
} 
into the host cell proper where they play a clear role in parasite virulence [6-8]. Dense granule proteins are secreted post-invasion and are found in the PV space and inserted into the PV membrane [3]. Although certain dense granule proteins are believed to be involved in purine salvage from host cells [9], the specific functions for most of these proteins or their importance to parasite survival remains unclear.

The post-translational modification of proteins by the addition of $\mathrm{N}$ and O-linked oligosaccharides is common in most eukaryotes studied to date. With few exceptions [10], these modifications are usually observed on proteins in the secretory pathway of cells. They are added onto proteins either during their translocation into the ER, in the case of N-linked oligosaccharides, or during their transport through the Golgi complex, in the case of the Olinked oligosaccharides. The protein-linked oligosaccharides are subsequently modified to various extents as they traverse the secretory pathway to their final destination. The type and the extent of these modifications vary from species to species and, within a given species, from tissue to tissue and during development. The protein-linked oligosaccharides have many important roles in eukaryotes. Within individual cells, these oligosaccharides and their modifications play a critical role in the correct folding of proteins and their export from the ER [11], and in the correct intracellular targeting of proteins [12]. Within the animal, the protein-linked oligosaccharides play diverse and important roles in all processes involving cellcell interactions [13]. Finally, protein-linked oligosaccharides also play important roles in the interaction of many viral, bacterial and protozoan pathogens with their host organisms [13].

Despite the importance of N-glycosylation of proteins in other eukaryotic genera, several studies have suggested that this post-translational modification is a rare event in apicomplexan parasites [14-17]. In spite of this, the apicomplexan genomes do appear to contain genes encoding at least a number of the core enzymes required for synthesis of the dolichol-linked precursor oligosaccharide as well as the Rft1 flippase and members of the OST complex [18]. However, the number of proteins where the presence of N-linked oligosaccharides has been demonstrated experimentally is limited to 2 in Toxoplasma [14,19], 2-3 in Plasmodium falciparum [17] and none in other apicomplexans. In the present study, we demonstrate that $\mathrm{N}$-glycosylation of proteins is actually very common in Toxoplasma gondii and that this modification is necessary for parasite growth. We also demonstrate that the structure of $\mathrm{N}$ linked oligosaccharides of Toxoplasma differs from that in other eukaryotes.

\section{Materials and Methods}

\subsection{Reagents and antibodies}

All reagents were purchased from either Fisher Scientific (Germantown, MD) or Sigma (St. Louis, MO) unless indicated otherwise. Monoclonal anti bodies to Gra1 (T52B4), Gra2 (T41F5), SAG2 (T43G11), MIC2 (T34A11), MIC3 (T42F3), gp23 (T42E12), and ROP8 (T52D1) were generous gifts of Dr. Jean Francois Dubremetz (Université de Montpellier, Montpellier, France). The SAG1 monoclonal antibody was purchased from Argene (North Massapequa, N.Y.). The generation of antisera to NTPase I and II has been described previously [9]. The monoclonal antibody HC10 was a generous gift of Dr. Frelinger (University of North Carolina, Chapel Hill, NC).

\subsection{Culture of host cells and Toxoplasma tachyzoites}

Toxoplasma gondii tachyzoites of the RH strain were maintained in confluent monolayers of human foreskin fibroblasts (HFF). The latter were maintained in $\alpha$-MEM medium with glutamine, penicillin and streptomycin (Invitrogen, Carlsbad, CA ) containing $2 \%$ fetal bovine serum (Hyclone, Logan, UT). Toxoplasma tachyzoites were maintained by serial passage on HFF cell monolayers every 2-3 days. 


\subsection{Tunicamycin treatment}

HFF monolayers to be used for experiments with tunicamycin were used at least 12 days after seeding $\sim 10^{5}$ freshly trypsinized cells per $\mathrm{cm}^{2}$. At this stage, the HFF cells were found to be entirely resistant to the effects of tunicamycin for at least 96 hours as judged by the following criteria: cell morphology, replating efficiency, Trypan blue exclusion, and a LIVE/DEAD Viability/Cytotoxicity Kit (Invitrogen, Carlsbad, CA). Tunicamycin titration were performed by incubating $2 \times 10^{5}$ parasites with confluent HFF monolayers in $25 \mathrm{~cm}^{2}$ flasks containing 4 $\mathrm{ml}$ complete medium with the indicated concentrations of tunicamycin or DMSO for 30 minutes, after which the medium was replaced with fresh medium containing the same concentration of tunicamycin or DMSO. After 2,24 , or 48 hours incubation at $37^{\circ} \mathrm{C}$, cells and parasites were collected by scraping, passed through an 18 and $21 \mathrm{G}$ needles to disrupt clumps, collected by centrifugation for 5 minutes at $2000 \mathrm{rpm}$, resuspended in $2 \mathrm{ml} \mathrm{PBS}$ and counted using a hemocytometer. All incubations were performed in triplicate.

Control or tunicamycin-treated parasites collected after 48 hours were subsequently tested for their ability to infect and replicate in fresh HFF monolayers in the same manner as described above.

The isolated parasites were also tested for their ability to perform gliding motility and to attach to and invade HFF cells as described before [22].

\subsection{Fluorescence and electron microscopy}

For fluorescence microscopy, free parasites attached to poly-L-lysine coated coverslips or infected HFF cells were fixed in cold $\left(-20^{\circ} \mathrm{C}\right)$ methanol, or in $3 \%$ paraformaldehyde in PBS followed by permeabilization in $0.5 \%$ Triton X-100 in PBS. Incubation with primary and secondary antibodies were performed as described [19]. For lectin overlays, fixed parasites or parasite-infected cells were incubate with biotinylated lectins (Vector Laboratories,

Burlingame, CA) diluted 1:500 in 3\% BSA in PBS followed by incubation with Texas Redconjugated streptavidin (Invitrogen) diluted 1:1000 in the same buffer. Images were captured using an epifluorescence Nikon Eclipse TE-2000 microscope (Nikon Inc., Melville, NY) and a Hamamatsu 1394 cooled digital CCD camera (Hamamatsu) and Metamorph ${ }^{\circledR}$ imaging software (Universal Imaging Corp.). Adobe Photoshop ${ }^{\circledR}$ (Adobe Systems Inc., San Jose, CA) was used to crop images.

For electron microscopy, Toxoplasma-infected HFF cells were washed twice in $0.1 \mathrm{M}$ sodium cacodylate $\mathrm{pH} 7.4$ and fixed overnight at $4{ }^{\circ} \mathrm{C}$ in $2 \%$ glutaraldehyde in $0.1 \mathrm{M}$ sodium cacodylate $\mathrm{pH}$ 7.4. After staining in $1 \%$ osmium tetroxide and dehydration in 50-100\% ethanol, the samples were embedded in epon. The polymerized bocks were sectioned at $60 \mathrm{~nm}$ with a Leica Ultracut UCT ultramicrotome (Leica Microsystems Inc., Bannockburn, IL). Sections were stained with 2\% uranyl acetate and Sato's lead stain, and viewed on an FEI Tecnai 12 electron microscope (FEI Company, Hillsboro, OR). Images were collected with a Gatan model 794 multi-scan digital camera (Gatan Inc., Pleasanton, CA).

\subsection{Lectin overlays}

Protein samples corresponding to $2 \times 10^{6}$ parasites or $5 \times 10^{5}$ HFF cells were separated by SDS-PAGE and transferred to nitrocellulose. This was blocked in 3\% BSA in Tris-buffered saline with $0.1 \%$ Tween 20 (TBST) and incubated for one hour with biotinylated concanavalinA or Dolichos biflorus agglutinin (Vector, Burlingame, CA) diluted 1:2000 and 1:1000 in TBST, respectively. Following incubation with IRDye800CW-conjugated streptavidin (LiCOR Biosciences, Lincoln, NE) the blots were scanned with a Li-COR Odyssey (Li-COR Biosciences). 
Where indicated, total parasite lysates were also digested prior to SDS-PAGE with PNGase F as suggested by the manufacturer (NEB).

\subsection{Metabolic labeling and immunoprecipitation}

For long-term labeling of proteins, Toxoplasma-infected or uninfected HFF cells (in $25 \mathrm{~cm}^{2}$ flasks) were washed twice with cysteine/methionine-free DMEM medium (Invitrogen) and incubated for $16-18$ hours at $37^{\circ} \mathrm{C}$ in $3 \mathrm{ml}$ cysteine /methionine-free DMEM medium containing $3 \%$ dialyzed fetal bovine serum and $358 \mu \mathrm{Ci}\left[{ }^{35} \mathrm{~S}\right]$ methionine/cysteine (Pro-Mix $\left[{ }^{35} \mathrm{~S}\right]$ in vitro cell labeling mix, GE Healthcare BioSciences Corp., Piscataway, NJ). Tunicamycin (EMD Biochemicals Inc., San Diego, CA) was added to $5 \mu \mathrm{g} / \mathrm{ml}$ where indicated. Cells were collected by scraping with a rubber policeman, collected by centrifugation for 5 minutes at $400 \times \mathrm{g}$, washed once in PBS and frozen in liquid nitrogen.

For pulse-chase experiments, Toxoplasma-infected or uninfected HFF cells were washed twice in cysteine/methionine-free DMEM and subsequently gently scraped into $2 \mathrm{ml}$ of the same medium using a rubber policeman. Cells were collected by centrifugation for 5 minutes at 400 $\times \mathrm{g}$, resuspended in $1 \mathrm{ml}$ cysteine/methionine-free DMEM containing $3 \%$ dialyzed fetal bovine serum, and incubated for 30 minutes at $37^{\circ} \mathrm{C}$. Pulse labeling was initiated by the addition of $358 \mu \mathrm{Ci}\left[{ }^{35} \mathrm{~S}\right]$ methionine/cysteine and incubated at $37^{\circ} \mathrm{C}$ for 10 minutes. Subsequently, methionine and cysteine were added to a final concentration of $0.1 \mathrm{mM}$. Samples $(0.45 \mathrm{ml})$ were removed, placed on ice, centrifuged for 1 minute at $14,000 \times \mathrm{g}$ and $4{ }^{\circ} \mathrm{C}$, and the resulting pellets frozen in liquid nitrogen. The remainder was incubated for an additional 60 minutes at $37^{\circ} \mathrm{C}$ after which $0.45 \mathrm{ml}$ was removed, centrifuged as described above and also frozen in liquid nitrogen.

Cell pellets were resuspended in $1 \%$ SDS in water and heated for 5 minutes at $95^{\circ} \mathrm{C}$. Samples were diluted by the addition of 9 volumes of $1 \%$ Triton X-100 in $150 \mathrm{mM} \mathrm{NaCl}, 5 \mathrm{mM}$ EDTA, $50 \mathrm{mM}$ Tris- $\mathrm{HCl} \mathrm{pH} 8$ (TNET) and insoluble material was removed by centrifugation for 10 minutes at $14,000 \times \mathrm{g}$ and $4^{\circ} \mathrm{C}$. GAP50 and HLA class I heavy chains were immunoprecipitated by the addition of a rabbit-anti-GAP50 antiserum and the HC10 monoclonal antibody [23]. After 60 minutes on ice, $25 \mu$ l of protein-A agarose (Zymed, S. San Francisco, CA) was added followed by an additional incubation for 30 minutes at $4^{\circ} \mathrm{C}$. Protein-A beads were washed three times in TNET buffer.

The immunoprecipitated material was treated where indicated with either endoglycosidase $\mathrm{H}$ or PNGase F (New England Biolabs, Beverly, MA) according to manufacturers' instructions.

Samples were analyzed by SDS-PAGE and fluorography with EN ${ }^{3}$ HANCE (Perkin Elmer, Boston, MA).

\section{Results}

\section{1. $\mathbf{N}$ and O-linked glycosylation of proteins is common in Toxoplasma gondii}

We previously observed that GAP50, the membrane anchor for the myosin XIV motor complex in Toxoplasma gondii, contains multiple N-linked oligosaccharides [19]. Previous studies by Schwarz and colleagues had demonstrated $\left[{ }^{3} \mathrm{H}\right]$ mannose incorporation into only a small number of Toxoplasma proteins, one of which was identified as the cell surface protein gp23 [14]. In light of these observations and in the context of devising alternative purification strategies for the myosin motor complex from Toxoplasma, we analyzed parasites by western blotting and fluorescence microscopy for the presence of proteins with $\mathrm{N}$-linked oligosaccharides using biotinylated lectins. Specifically, we used the mannose-specific lectin concanavalin-A (con-A) to detect $\mathrm{N}$-glycosylated proteins and the $\mathrm{N}$-acetylgalactosaminespecific lectin from Dolichos biflorus lectin (DBA) to detect O-glycosylated proteins. 
Total Toxoplasma tachyzoite lysates were fractionated by SDS-PAGE, transferred to nitrocellulose, and incubated with the biotinylated lectins. Host cell lysates were analyzed in parallel to control for possible contamination of the parasite preparation. We also compared reactivity of lectins with untreated and PNGase F-treated Toxoplasma protein preparations to ensure that all reactivity was due to the presence of $\mathrm{N}$-linked oligosaccharides. To our surprise, we found that Toxoplasma tachyzoites contained a large number of proteins reactive with con$\mathrm{A}$ and DBA, indicating that $\mathrm{N}$ and $\mathrm{O}$-glycosylation of proteins is as common in $T$. gondii as it is in other eukaryotes (Fig. 1). Based on the number of protein species reactive with con-A, we estimate that Toxoplasma tachyzoites contain at least 11 major N-glycosylated proteins and multiple minor ones. The reactivity of con-A with the parasite proteins is due to the presence of N-linked oligosaccharides on these proteins as it is absent in parasite lysates pretreated with the N-glycan specific enzyme PNGase F (Fig. 1). The absence of labeling is not due to degradation or loss of protein as the PNGaseF treated and non-treated samples contained similar quantities of the membrane skeleton protein IMC1, the myosin light chain MLC1, and GAP50. As the latter is known to be $\mathrm{N}$-glycosylated, its decrease in $\mathrm{MW}_{\text {app }}$ upon PNGase $\mathrm{F}$ digestion serves as an internal control for this enzyme. Based on a similar analysis with DBA, we estimate that more than 15 Toxoplasma proteins contain O-linked oligosaccharides exist in Toxoplasma. The reactivity of DBA with Toxoplasma proteins was, as expected, not affected by PNGase F treatment (Fig. 1). Surprisingly, the reactivity of DBA with Toxoplasma proteins was not affected by digestion with O-glycanase (data not shown). Incubation of parasite proteins with anhydrous trifluoromethanesulfonic acid, a treatment that is used to hydrolyze both $\mathrm{N}$ and O-linked oligosaccharides, also did not affect reactivity with DBA under conditions where all reactivity with con-A was lost (data not shown).

The observations were found to be very consistent between different parasite preparations and therefore indicate that both $\mathrm{N}$ and O-linked glycosylation of proteins in $T$. gondii is far more common than was previously believed.

\section{2. $\mathrm{N}$ and $\mathrm{O}-\mathrm{glyc}$ cosylated proteins are found throughout parasite}

As stated above, the majority of $\mathrm{N}$ and $\mathrm{O}$-glycosylated proteins in other eukaryotes are found along the secretory and endocytic/phagocytic pathways, on the plasma membrane and in the surrounding medium. In order to determine the general location of $\mathrm{N}$ and $\mathrm{O}$-glycosylated proteins in Toxoplasma tachyzoites, we probed fixed extracellular parasites with the various biotinylated lectins used in Fig. 1, followed by a Texas Red-conjugated streptavidin. We observed con-A-reactive material in multiple structures throughout extracellular parasites that overlapped to various extents with all markers for the secretory pathway (Supplementary Fig. 1). It was impossible to determine if any of the con-A reactive proteins of Toxoplasma were secreted into the parasitophorous vacuole surrounding intracellular parasites as binding of this lectin to host cell glycoproteins effectively obscures any interaction with parasite glycoproteins. DBA-reactive material appeared to be confined to structures found between the parasite nucleus and the anterior end of the parasites. These DBA-positive structures did not obviously co-localize with any makers of known secretary organelles in Toxoplasma suggesting they may represent structures that have not been characterized to date (data not shown). Together with the data shown in Fig. 1, these data demonstrate that Toxoplasma synthesizes a large number of proteins with $\mathrm{N}$ and O-linked glycans that are found throughout the secretory pathway of the parasite.

\section{3. $\mathrm{N}$-glycosylation is essential for Toxoplasma viability}

The data in the previous sections demonstrate that $\mathrm{N}$-linked glycosylation is a common posttranslational modification of Toxoplasma proteins. To determine if $\mathrm{N}$-glycosylation of proteins is as critical to Toxoplasma survival as it is in other eukaryotes, we tested the effect of tunicamycin on protein glycosylation and parasite growth. 
We monitored the effect of tunicamycin on N-glycosylation of proteins in Toxoplasma using GAP50, the only characterized N-glycosylated protein in the parasite. GAP50 was immunoprecipitated from parasites that had been metabolically labeled with $\left[{ }^{35} \mathrm{~S}\right]$ methionine in the absence or presence of $5 \mu \mathrm{g} / \mathrm{ml}$ tunicamycin, a concentration that was sufficient to block glycosylation of HLA class I antigens in uninfected HFF cells (not shown). As can be seen in Fig. 2, this concentration of tunicamycin also suffices to block N-glycosylation of GAP50 completely.

Treatment of other cells types with tunicamycin typically results in the accumulation of nonglycosylated and unfolded proteins in the endoplasmic reticulum and cell death [20]. Completely confluent HFF cells, in sharp contrast to non-confluent cultures, were not obviously affected by treatment with up to $20 \mu \mathrm{g} / \mathrm{ml}$ tunicamycin for 72 hours as judged by intactness of the monolayer, cell morphology, cell integrity (as judged by trypan blue exclusion and a LIVE/DEAD cell viability assay), and cell viability (Supplementary Fig. 2). As Toxoplasma infection and replication was not affected by HFF cell density, the use of confluent HFF cells allowed us to determine the long-term effect of tunicamycin on Toxoplasma replication and morphology.

Treatment of Toxoplasma-infected, confluent HFF cultures with $5 \mu \mathrm{g} / \mathrm{ml}$ tunicamycin resulted in the complete inhibition of GAP50 N-glycosylation as judged by immunoblot (Fig. 3A) confirming that the $\mathrm{N}$-glycosylation machinery of Toxoplasma is sensitive to tunicamycin under these experimental conditions. To determine how long-term inhibition of $\mathrm{N}$ glycosylation affects Toxoplasma viability and structure, we infected confluent HFF monolayers with Toxoplasma and treated these with different concentrations of tunicamycin. A typical Toxoplasma infection cycle, from the initial infection through parasite egress, lasts 48 hours in HFF cells. As we did not know the kinetics with which tunicamycin affects the parasite, we performed the tunicamycin titrations over a total period of 96 hours. This was divided into two 48 hour infection cycles in which parasites obtained from the first 48 hour cycle (stage 1) were used to re-infect fresh HFF monolayers and followed for an additional 48 hours (stage 2). Tunicamycin concentrations were maintained at identical levels in both stages. Parasites were harvested at different time points and counted.

During the first 48 hour infection cycle (Stage 1, Fig. 3B), treatment with increasing concentrations of tunicamycin resulted in a decreased parasite yield. The maximal effect was observed with $5 \mu \mathrm{g} / \mathrm{ml}$ tunicamycin but even at higher concentrations we did not observe a complete inhibition of parasite replication. This was a surprising finding in that inhibition of glycosylation was complete at these concentrations (Fig. 3A). In stage 2 of this experiment, we determined if the parasites that had been grown for 48 hours in various tunicamycin concentrations in stage 1 were still capable of host cell infection and replication. As can be seen in figure $3 \mathrm{~B}$, parasites that were grown in the presence of as little as $5 \mu \mathrm{g} / \mathrm{ml}$ tunicamycin in stage 1 were incapable of growth in new cultures during stage 2.

This observation could be explained by tunicamycin inducing more or less specific defects in parasite motility, host cell attachment or invasion. As can be seen in Figs. 3C and 3D,

tunicamycin-treated Toxoplasma tachyzoites appear to be severely defective in all three assays. A small fraction of the tunicamycin-treated parasites did infect the new monolayer in stage 2 of the experiment but the vast majority ( $>95 \%$ ) failed to replicate. The few that did replicate only gave rise to small vacuoles containing 4 or less parasites. Treatment of Toxoplasma with $10 \mu \mathrm{g} / \mathrm{ml}$ tunicamycin for 48 hours does not result in any obvious changes in either the expression level or distribution of GAP50, the one well-characterized N-glycosylated protein in Toxoplasma (Fig. 4). Similarly, we did not observe obvious effects on the amount or localization of marker proteins for the parasite plasma membrane, the endoplasmic reticulum, or the three major late secretory organelles: the rhoptries, dense granules, and micronemes 
(Supplementary Fig 1B). The apicoplast, a plastid-like organelle embedded in the secretory apparatus of apicomplexan parasites, also did not appear to be affected as judged by staining of the organellar DNA with DAPI. The same staining also revealed no obvious effects of the tunicamycin treatment on nuclear morphology.

As indicated above, treatment of Toxoplasma tachyzoites with tunicamycin for 48 hours renders the parasites largely incapable of invading into new host cells. When the few parasites that had invaded were analyzed after an additional 48 hours of tunicamycin treatment, effects on parasite morphology, protein expression, and protein distribution were more pronounced. Expression of GAP50 and the dense granule protein GRA3 were greatly diminished compared to untreated parasites (Fig. 4, Supplementary Fig. 3). The expression level of the plasma membrane protein SAG1 and the microneme protein MIC2 was not noticeably affected but marked intracellular accumulations of both proteins were evident (Supplementary Fig. 3A). In the case of MIC2, the accumulation appeared to occur in the nuclear envelope and endoplasmic reticulum. SAG1, on the other hand, accumulated in anterior structures that did not contain marker proteins for any of the known secretory organelles in the parasite. Tunicamycin treatment did not have a noticeable impact on either the expression level or transport of either Toxoplasma BiP or the rhoptry protein ROP2. Nuclear morphology was clearly affected in the vast majority of parasites as was the frequency with which DAPI-positive apicoplasts were observed. Unlike what is seen in animal cells, tunicamycin treatment did not induce condensed nuclei in Toxoplasma.

We used transmission electron microscopy to analyze the effect of tunicamycin treatment on the subcellular structure of Toxoplasma tachyzoites (Fig. 5). After 48 hours treatment with 10 $\mu \mathrm{g} / \mathrm{ml}$ tunicamycin, we did observed only one reproducible structural effect on Toxoplasma in that the nuclear envelope of tunicamycin-treated parasites consistently appeared slightly distended (Fig. 5B, inset). After 96 hours, the impact of tunicamycin treatment was obvious, with an extensive distension of the nuclear envelope and endoplasmic reticulum, and an absence of recognizable dense granules and rhoptries (Fig. 5C). The latter observation indicates that the ROP2-positive structures observed by light microscopy either do not represent normal rhoptries or that the structures of these organelles has changed beyond recognition. A defined nucleus was also absent from the parasites at this stage which is consistent with the light microscopic observations in Supplementary Fig. 3.

\subsection{Other $\mathrm{N}$-glycosylated proteins of Toxoplasma}

In the experiments described above, we used the integral membrane protein GAP50 to monitor the effectiveness with which tunicamycin blocks N-glycosylation in Toxoplasma tachyzoites. As the data in Fig. 1 indicate, multiple N-glycosylated proteins exist in these organisms. The report by Odenthal-Schnittler et al [14] indicates that gp23 may be one of these, although we did not observe an obvious conA-reactive glycoprotein in the $23 \mathrm{kDa}$ molecular weight range (Fig. 1A). When we analyzed gp23 directly for the presence of $\mathrm{N}$-linked oligosaccharides by PNGase F digestion, however, we did not observe any noticeable change in its $\mathrm{MW}_{\text {app }}$ in SDSPAGE (Supplementary Fig. 4A). The $\mathrm{MW}_{\text {app }}$ was also not noticeably affected in tunicamycintreated parasites (data not shown).

To identify other potential N-glycosylated Toxoplasma proteins, we analyzed predicted protein sequences generated form the Toxoplasma genome project (toxodb.org) for the presence of additional candidate $\mathrm{N}$-glycosylated proteins. Specifically, we searched the database for protein sequences predicted to possess $\mathrm{N}$-terminal signal sequences and this subset was subsequently analyzed for candidates that had consensus N-glycosylation sites (N-X-S/T, where $\mathrm{X}$ is not a proline). Multiple candidates were identified using this strategy including a number of known Toxoplasma secretory or plasma membrane proteins (Supplementary table 1). We analyzed a number of these candidates for the presence of N-linked oligosaccharides 
by comparing their $\mathrm{MW}_{\text {app }}$ before and after digestion with PNGase F. As can be seen in Supplementary table 1 and Supplementary Fig. 4B, none of the candidate proteins appeared $\mathrm{N}$-glycosylated based on this criterion. This observation suggests that, apart from GAP50, the Toxoplasma glycoproteins identified in Fig. 1 may represent novel parasite proteins. We have initiated the identification of these proteins using mass spectroscopy of lectin-affinity purified parasite proteins.

\section{5. $\mathbf{N}$-linked oligosaccharides of Toxoplasma differ from those in other eukaryotes}

In most eukaryotes, the N-linked oligosaccharides on proteins exported from the ER are modified extensively through the addition and/or removal of sugar residues or their derivatives. Typically, this results in noticeable alteration in the apparent molecular weight ( $\mathrm{MW}_{\mathrm{app}}$ ) of these proteins during SDS-PAGE. We analyzed whether there were any major alterations in the $\mathrm{MW}_{\text {app }}$ of GAP50 during a pulse-chase experiment. As can be seen in Fig. 6, there is no obvious change in the motility of GAP50 in this experiment suggesting that, although processing the $\mathrm{N}$-linked glycans may be occurring during the experiment, these are not substantial enough to alter the $\mathrm{MW}_{\mathrm{app}}$ of GAP50.

A commonly used method to observe at least part of N-linked glycan processing of a protein during its intracellular transport from the ER to the Golgi complex is to incubate the glycoprotein with the enzyme endoglycosidase $\mathrm{H}(\mathrm{endoH})$. Whereas the $\mathrm{N}$-linked oligosaccharides found on glycoproteins in the ER are sensitive to cleavage by this enzyme, processing of the oligosaccharide in the Golgi complex renders it endoH-resistant. This is demonstrated in the control experiment shown in Fig. 6, were we analyzed the endoHsensitivity of the N-linked glycans on HLA class I heavy chain that was immunoprecipitated in parallel from host cells. When we analyzed whether there was a similar change in the sensitivity of N-linked glycans on GAP50 to endoH, we found to our surprise that these were endoH-resistant at all times. This effect is not due to their inaccessibility to endoH as the oligosaccharides were readily removed by PNGase F, a glycosidase that removes all N-linked glycans from proteins. Taken together with our previous observations these data indicate that $\mathrm{N}$-glycosylation is a common post-translational modification of proteins in Toxoplasma gondii but that the structure of these glycans differs substantially from that encountered to date in animals, plants, and other unicellular organisms.

\section{Discussion}

Protein glycosylation is a common post-translational modification of proteins in all eukaryotic cells studied to date and is known to play important roles in the quality control of protein folding in the endoplasmic reticulum, in the control of intracellular trafficking of proteins, and in the regulation of cell-cell and cell-substrate interactions. Protein glycosylation also plays a critical role in the interaction of various pathogens with their host cells [13]. Despite this, neither the existence of N-glycosylation in Toxoplasma nor its role in the parasite's cellular processes or ability to cause disease has received a lot of attention beyond the initial reports by Schwarz and co-workers $[14,15]$. As a result of this, little consideration has been given to the possible role of Toxoplasma $\mathrm{N}$-linked glycans in the efficacy of potential vaccine candidates or in the immune response to Toxoplasma in general.

A careful analysis by Samuelson and colleagues [18] of the genomic and EST sequences obtained in the Toxoplasma genome project indicates that Toxoplasma, and its close relatives Cryptosporidium and the malaria parasite Plasmodium, do in fact possess the general enzymatic machinery to synthesize dolichol-linked precursors for the N-linked oligosaccharides as well as the machinery to transfer it to nascent polypeptide chains. Their analysis did not result in the identification of homologues for all of the enzymes known to be involved in $\mathrm{N}$-glycan synthesis in other eukaryotes. Whether this reflects a true absence of 
these enzyme activities in the parasites, a lack of their evolutionary conservation or the incomplete nature of the databases remains to be determined. We have repeated the analysis performed by Samuelson et al using more recent versions of the Toxoplasma sequence databases. Our analysis largely confirms their findings and in fact gave rise to the identification of a homolog for only one additional enzyme (Supplementary Fig. 5).

Our lectin overlay experiments suggest that Toxoplasma tachyzoites contain at least 11 major and multiple minor N-glycosylated proteins (Fig. 1). Thus far, only two Toxoplasma proteins have been reported to be N-glycosylated. The presence of N-linked glycans on GAP50, the membrane anchor for the myosin XIV motor complex in the parasite, was demonstrated through digestion with PNGase F and through its sensitivity to tunicamycin. (Figs. 1 and 2). The other protein, gp23, is a GPI-anchored Toxoplasma surface protein which has not been characterized by molecular cloning [14]. The presence of N-linked oligosaccharides on this protein was based on the incorporation of radiolabeled sugars and their release by PNGase F [14]. The authors of that work also found that the radiolabeled oligosaccharides were resistant to endo $\mathrm{H}$, but interpreted this to mean that, like in higher eukaryotes, the oligosaccharides had matured to a complex type structure. In the light of our findings and the analysis by Samuelson et al, it is also possible that the gp23 oligosaccharides are endoH-resistant because their structure lacks the prerequisite mannose residue. In our hands, gp23 did not appear to be affected by treatment with tunicamycin or PNGase F, however. Further analysis of gp23 is needed to address these discrepancies.

Like all in other eukaryote cells studied to date, N-liked glycosylation of proteins is essential for the survival of Toxoplasma. Treatment of the parasite with tunicamycin results in parasite death although this occurs with unusual kinetics. When extracellular or intracellular Toxoplasma tachyzoites are treated with tunicamycin, $\mathrm{N}$-glycosylation is immediately inhibited (Fig. 2). This does not result in an immediate block in the ability of the parasites to invade into host cells, but their ensuing replication is blocked by more than $950 \%$ (Fig. 3B). Apart from having a slightly distended nuclear envelope the resulting parasites appear normal at the ultrastructural level and their major secretory organelles do not appear affected (Fig. 5B). These parasites are almost completely incapable of invading new host cells, however (Fig. 3B). The few parasites that do invade replicate only once or twice and accumulate with deformed nuclei, vastly distended endoplasmic reticulum and nuclear envelope, and without recognizable late secretory organelles (Fig. 4, 5C and Supplementary Fig. 3).

The latter observations are consistent with the general disruption of the secretory pathways typically observed in other eukaryotes [12]. There are a number of possible explanations for the observation that disruption of $\mathrm{N}$-glycosylation by tunicamycin does not result in a large effect on parasite ultrastructure or replication rate during the first 48 hours of incubation, but a very substantial effect on the subsequent motility and host cell invasion. Our data are most consistent with a model in which most Toxoplasma glycoproteins are not important for parasite growth or replication inside animal host cells, but are critical for the processes that follow their release from these cells: motility and host cell invasion.

The analysis of the Toxoplasma genome for the presence of the various enzymes (ALG protein homologs) known to be required for the synthesis of the dolichol-linked precursor oligosaccharide suggests that the parasite may not be able to synthesize the structure typically encountered in animal cells and yeasts (Supplementary Fig. 5, Samuelson et al [18], this study). Specifically, homologs of the genes encoding ALG3p (dolichyl-P-Man:Man ${ }_{5} \mathrm{GlcNAc}_{2}-\mathrm{PP}-$ dolichyl $\alpha$ (1-3)-mannosyltransferase), ALG9p (dolichyl-P-Man:Man ${ }_{6} \mathrm{GlcNAc}_{2}-\mathrm{PP}-$ dolichyl $\alpha(1-2)$-mannosyltransferase) and ALG12p (dolichyl-P-Man:Man ${ }_{7}$ GlcNAc$_{2}$-PP-dolichyl- $\alpha$ (1-6)-mannosyltransferase) appear to be lacking from the Toxoplasma genome (Supplementary Fig. 5). As the glucosyltransferase responsible for the addition of the three terminal glucose 
residues to the precursor oligosaccharide are present in the parasite genome, it would suggest that N-glycosylation of proteins in Toxoplasma would involve the structure shown in Fig. 7. The absence of gene products with homology to known enzymes from Toxoplasma does not prove, however, that a particular enzyme activity is actually absent as it may be supplied by a structurally unrelated, parasite-specific enzyme. The experiments shown in Fig. 6 demonstrate that this is unlikely to be the case. Endoglycosidase H requires Man- $\alpha(1-3)-M a n-\alpha(1-6)-M a n-$ $\beta(1-4)$-GlcNAc- $\beta(1-4)$-GlcNAc-Asn as minimal structure in order to cleave the N-linked oligosaccharide from a glycoprotein [21]. The observation that the Toxoplasma glycoprotein is always resistant to the action of endoglycosidase $\mathrm{H}$, but not PNGase $\mathrm{F}$, indicates that this particular mannose is indeed absent from Toxoplasma N-linked oligosaccharides, supporting the notion that an ALG3-like enzyme activity is absent from the parasite.

We have not addressed the presence of N-linked oligosaccharides on protein in Plasmodium and Cryptosporidium species at this time. Whereas the Cryptosporidium genome is predicted to encode homologs of the same ALG genes present in Toxoplasma and is therefore likely to possess similar N-linked oligosaccharides( [18] and Supplementary Fig. 5). This is not the case in Plasmodium species where the genome does not appear to encode any of ALG gene homologs except for ALG7 [18]. There have been conflicting reports on the presence of Nlinked oligosaccharides on Plasmodium proteins. Dieckmann-Schnittler et al could not detect any evidence for the presence of ALG7 activity in P. falciparum lysates or oligosaccharide transferase activity, nor could they detect any evidence for sensitivity to tunicamycin [16]. Although this would appear to be in conflict with the analysis of Samuelson et al, it is important to remember that biochemical analyses of Plasmodium extracts is complicated by the small yield and by the extensive contamination by host cell material. In contrast to these observations, Kimura et al reported that replication of $P$. falciparum is moderately affected by the presence of tunicamycin [17] and that a small number of con-A reactive proteins are present in parasiteinfected erythrocytes. The latter observation is surprising in that the analysis by Samuelson et al suggests that the Plasmodium genome does not posses genes encoding the mannosyl transferases necessary to generate a con-A-reactive high mannose oligosaccharide are absent [18].

Our studies indicate that $\mathrm{N}$-glycosylation of proteins is a common post-translational modification in Toxoplasma and that the structure of the oligosaccharides lacks a number of the elements typically encountered in other species. We do not know, however, the identity or location of the N-glycosylated proteins other than GAP50, nor do we know the extent to which the N-linked oligosaccharides are modified post-translationally. Considering the clinical importance of Toxoplasma and related apicomplexan parasites like Plasmodium and Cryptosporidium it is evident that further study of $\mathrm{N}$-glycosylation in these organisms may yield novel diagnostic and therapeutic strategies. The analysis of N-linked oligosaccharide synthesis and structure in these parasites is also likely to shed more light on the role of this post-translational modification in general.

\section{Supplementary Material}

Refer to Web version on PubMed Central for supplementary material.

\section{Acknowledgements}

The authors would like to thank Stacey Gilk and Sebastien Pomel for critical reading of the manuscript and Jean Francois Dubremetz for monoclonal antibodies to the various Toxoplasma proteins.

\section{Abbreviations}

PNGase F, Peptide: N-Glycosidase F. 


\section{References}

1. Dubey JP. Toxoplasmosis. J Am Vet Med Assn 1994;205:1593-98.

2. Wong SY, Remington JS. Toxoplasmosis in Pregnancy. Clin Inf Dis 1994;18:853-61.

3. Lingelbach K, Joiner KA. The parasitophorous vacuole membrane surrounding Plasmodium and Toxoplasma: An unusual compartment in infected cells. J Cell Sci 1998;111:1467-75. [PubMed: 9580555]

4. Carruthers VB. Proteolysis and Toxoplasma invasion. Int J Parasitol 2006;36:595-600. [PubMed: 16600244]

5. Beckers CJ, Dubremetz JF, Mercereau PO, Joiner KA. The Toxoplasma gondii rhoptry protein ROP 2 is inserted into the parasitophorous vacuole membrane, surrounding the intracellular parasite, and is exposed to the host cell cytoplasm. J Cell Biol 1994;127:947-61. [PubMed: 7962077]

6. Saeij JPJ, Boyle JP, Coller S, Taylor S, Sibley LD, Brooke-Powell ET, Ajioka JW, Boothroyd JC. Polymorphic secreted kinases are key virulence factors in toxoplasmosis. Science 2006;314:1780-3. [PubMed: 17170306]

7. Saeij JPJ, Coller S, Boyle JP, Jerome ME, White MW, Boothroyd JC. Toxoplasma co-opts host gene expression by injection of a polymorphic kinase homologue. Nature 2007;445:324-7. [PubMed: 17183270]

8. Taylor S, Barragan A, Su C, Fux B, Fentress SJ, Tang K, Beatty WL, El Hajj H, Jerome M, Behnke MS, et al. A secreted serine-threonine kinase determines virulence in the eukaryotic pathogen Toxoplasma gondii. Science 2006;314:1776-80. [PubMed: 17170305]

9. Bermudes D, Peck KR, Afifi MA, Beckers CJ, Joiner KA. Tandemly repeated genes encode nucleoside triphosphate hydrolase isoforms secreted into the parasitophorous vacuole of Toxoplasma gondii. $\mathrm{J}$ Biol Chem 1994;269:29252-60. [PubMed: 7961894]

10. Zachara NE, Hart GW. Cell signaling, the essential role of O-GlcNAc. Biochim. Biophys. Acta 2006;1761:599-617. [PubMed: 16781888]

11. Helenius A, Aebi M. Roles of N-linked glycans in the endoplasmic reticulum. Ann Rev Biochem 2004;73:1019-49. [PubMed: 15189166]

12. Varki A. Biological roles of oligosaccharides: all of the theories are correct. Glycobiology 1993;3:97130. [PubMed: 8490246]

13. Varki, A.; Cummings, R.; Esko, J.; Freeze, H.; Hart, GW.; Marth, J., editors. Essentials of Glycobiology. Cold Spring Harbor Laboratory Press; Cold Spring Harbor: 1999.

14. Odenthal-Schnittler M, Tomavo S, Becker D, Dubremetz JF, Schwarz RT. Evidence for N-linked glycosylation in Toxoplasma gondii. Biochem J 1993;291:713-21. [PubMed: 8489499]

15. Dieckmann-Schuppert A, Bause E, Schwarz RT. Glycosylation reactions in Plasmodium falciparum, Toxoplasma gondii, and Trypanosoma brucei brucei probed by the use of synthetic peptides. Biochim Biophys Acta 1994;1199:37-44. [PubMed: 8280751]

16. Dieckmann-Schuppert A, Bender S, Odenthalschnittler M, Bause E, Schwarz RT. Apparent lack of $\mathrm{N}$-glycosylation in the asexual intraerythrocyticstage of Plasmodium falciparum. Eur J Biochem 1992;205:815-25. [PubMed: 1374032]

17. Kimura EA, Couto AS, Peres VJ, Casal OL, Katzin AM. N-linked glycoproteins are related to schizogony of the intraerythrocytic stage in Plasmodium falciparum. J Biol Chem 1996;271:1445261. [PubMed: 8662869]

18. Samuelson J, Banerjee S, Magnelli P, Cui J, Kelleher DJ, Gilmore R, Robbins PW. The diversity of dolichol-linked precursors to Asn-linked glycans likely results from secondary loss of sets of glycosyltransferases. Proc Natl Acad Sci USA 2005;102:1548-53. [PubMed: 15665075]

19. Gaskins E, Gilk S, DeVore N, Mann T, Ward G, Beckers C. Identification of the membrane receptor of a class XIV myosin in Toxoplasma gondii. J. Cell Biol 2004;165:383-93. [PubMed: 15123738]

20. Patil C, Walter P. Intracellular signaling from the endoplasmic reticulum to the nucleus: the unfolded protein response in yeast and mammals. Curr Op Cell Biol 2001;13:349-55. [PubMed: 11343907]

21. Maley F, Trimble RB, Tarentino AL, Plummer TH. Characterization of glycoproteins and their associated oligosaccharides through the use of endoglycosidases. Anal Biochem 1989;180:195-204. [PubMed: 2510544] 
22. Kieschnick H, Wakefield T, Narducci CA, Beckers C. Toxoplasma gondii attachment to host cells is regulated by a calmodulin-like domain protein kinase. J. Biol. Chem 2001;276:12369-77. [PubMed: 11154702]

23. Stam N, Spits H, Ploegh H. Monoclonal antibodies raised against denatured HLA-B locus heavy chains permit biochemical characterization of certain HLA-C locus products. J Immunol 1986;137:2299-306. [PubMed: 3760563] 
A

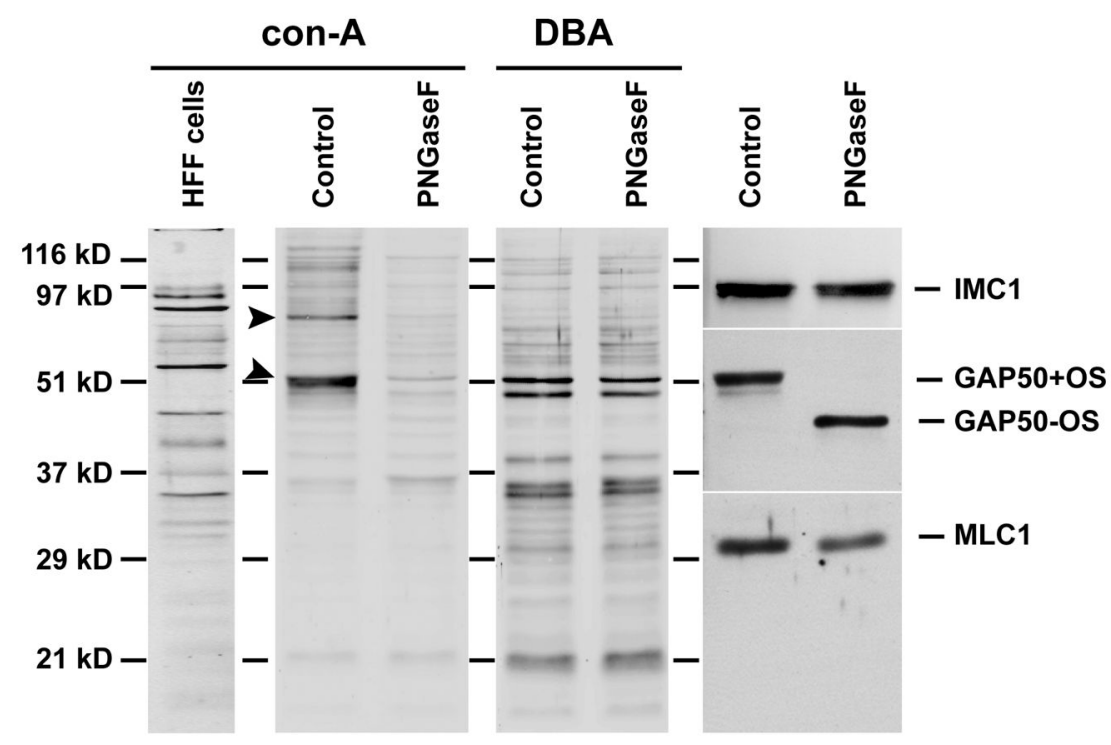

B

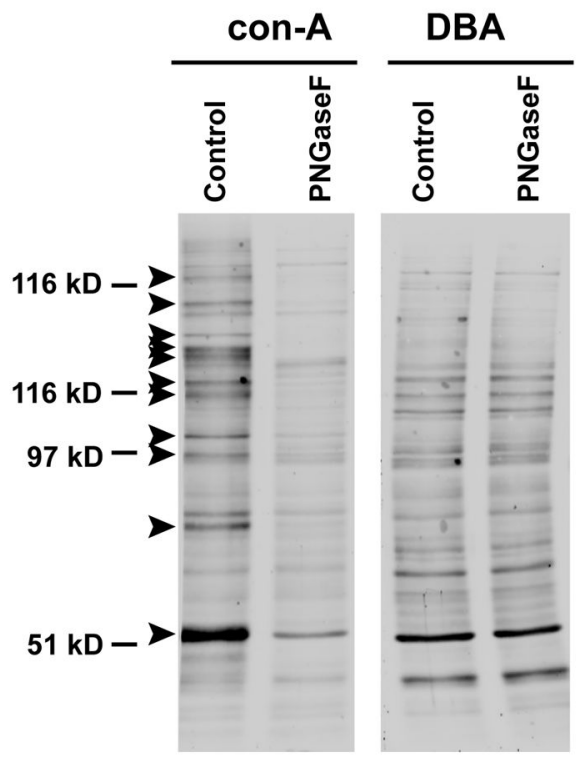

Figure 1.

$\mathrm{N}$ and O-glycosylation of proteins are common post-translational modifications in Toxoplasma tachyzoites.

Lysates of purified tachyzoites were incubated in the absence (Control) or presence (PNGase F) of PNGase $\mathrm{F}$ for 16 hours at $37^{\circ} \mathrm{C}$, fractionated by SDS-PAGE on $12 \%$ (A) or $7.5 \%$ (B) gels, and transferred to nitrocellulose. Blots were probed with biotinylated lectins concanavalin-A (con-A) and Dolichos biflorus agglutinin (DBA), and with monospecific antisera to the Toxoplasma glycoprotein GAP50 and the cytoplasmic proteins MLC1 and IMC1. Arrowheads indicate the con-A-reactive parasite proteins that are PNGase F sensitive. GAP50+OS and GAP50-OS refer the fully N-glycosylated and enzymatically deglycosylated GAP50, respectively. 

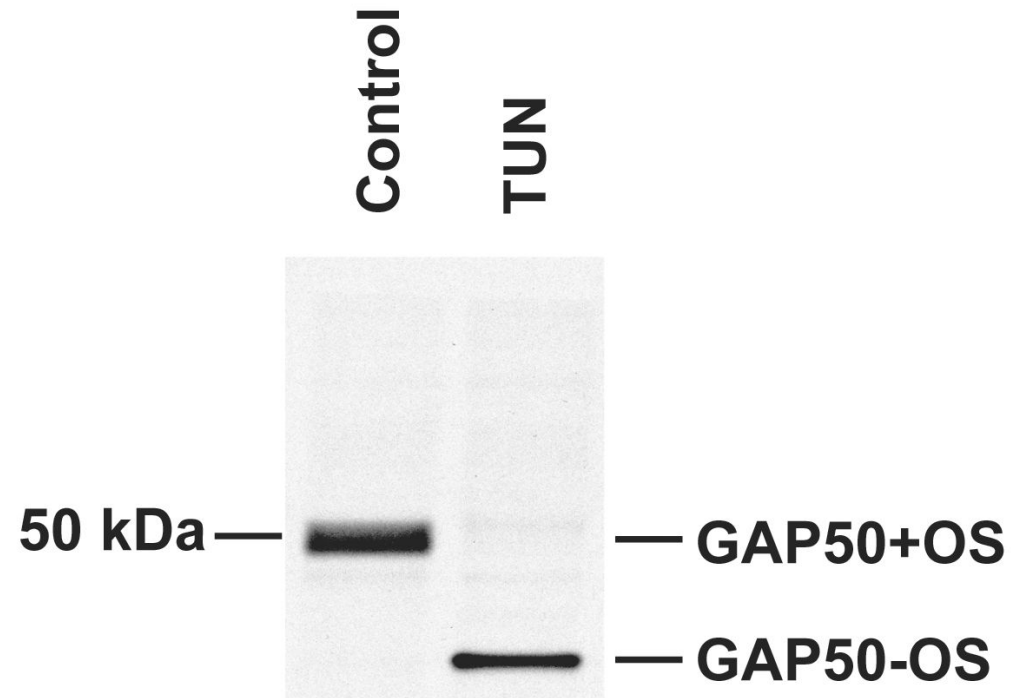

\section{$37 \mathrm{kDa}-$}

Figure 2.

$\mathrm{N}$-glycosylation in Toxoplasma gondii is sensitive to tunicamycin.

GAP50 was immunoprecipitated from Toxoplasma-infected HFF cells that were labeled for 16 hours with $\left[{ }^{35} \mathrm{~S}\right]$ methionine/cysteine in the presence or absence of $5 \mu \mathrm{g} / \mathrm{ml}$ tunicamycin. GAP50+OS and GAP50-OS refer to fully N-glycosylated and non-glycosylated GAP50, respectively. 

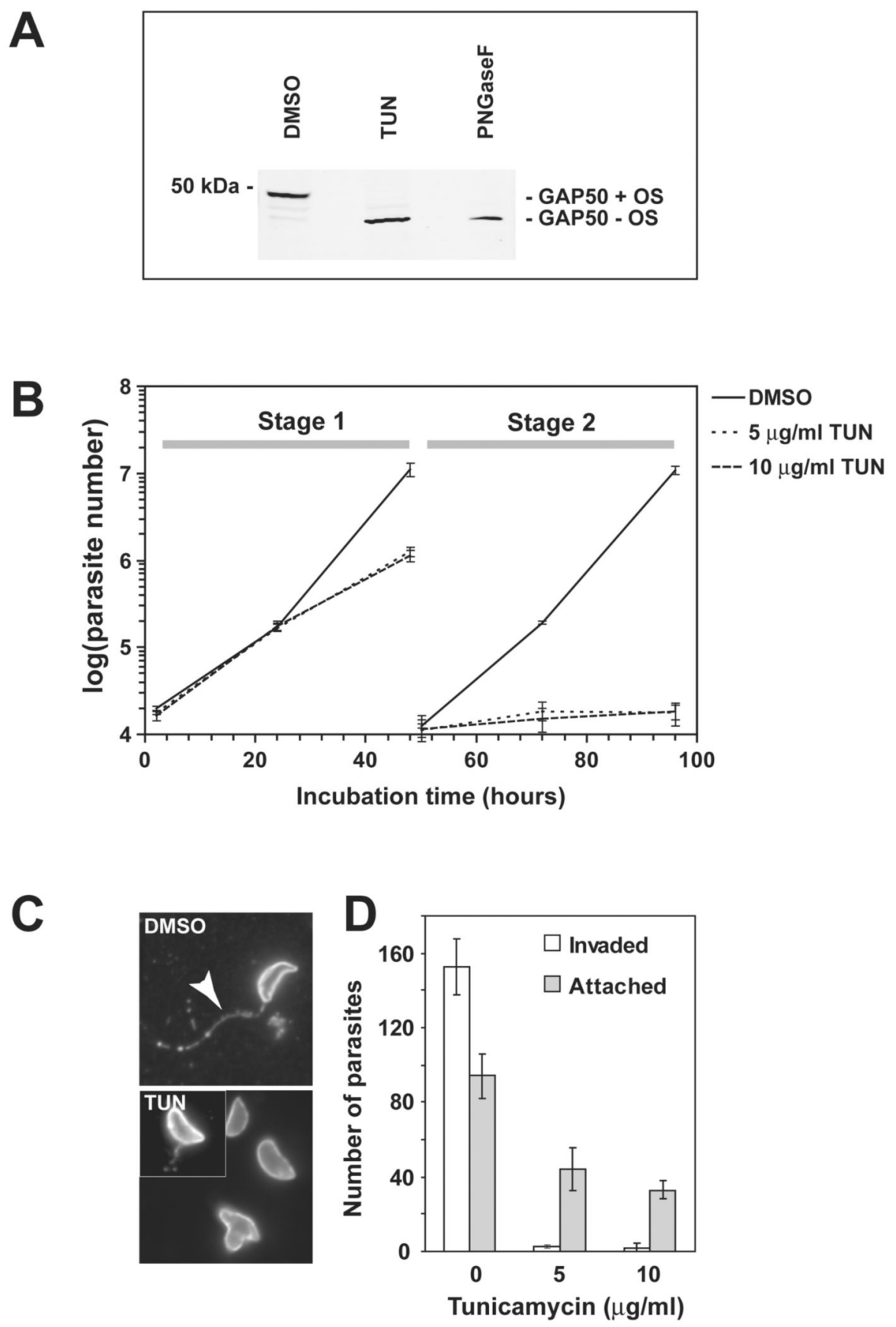

Figure 3.

N-glycosylation is critical for Toxoplasma survival.

A) GAP50 accumulates in non-glycosylated form after growth of Toxoplasma for 24 hours in the presence of $5 \mu \mathrm{gr} / \mathrm{ml}$ tunicamycin. GAP50+OS and GAP50-OS refer to fully N-

glycosylated and non-glycosylated GAP50, respectively.

B) The effect of tunicamycin on Toxoplasma cell viability was determined in a two-stage experiment. In stage 1, confluent HFF monolayers were infected with freshly isolated parasites in the presence of the indicated concentration of tunicamycin or DMSO. Unattached parasites were removed by washing after 2 hours at $37^{\circ} \mathrm{C}$ and the incubation continued in the presence 
or absence of tunicamycin. The number of host cell-associated parasites was determined at the indicated times.

In stage 2, parasites that had been isolated after 48 hours in the presence or absence of tunicamycin and used to infect fresh confluent HFF monolayers. Parasite replication was monitored in the same fashion as in stage 1.

C) Parasites treated for 48 hours with $5 \mu \mathrm{g} / \mathrm{ml}$ tunicamycin are severely defective in gliding motility. The arrowhead indicates the trail of cell surface protein left behind by the moving parasite.

D) Tunicamycin-treated (48 hours) parasites are defective in host cell attachment and invasion. 

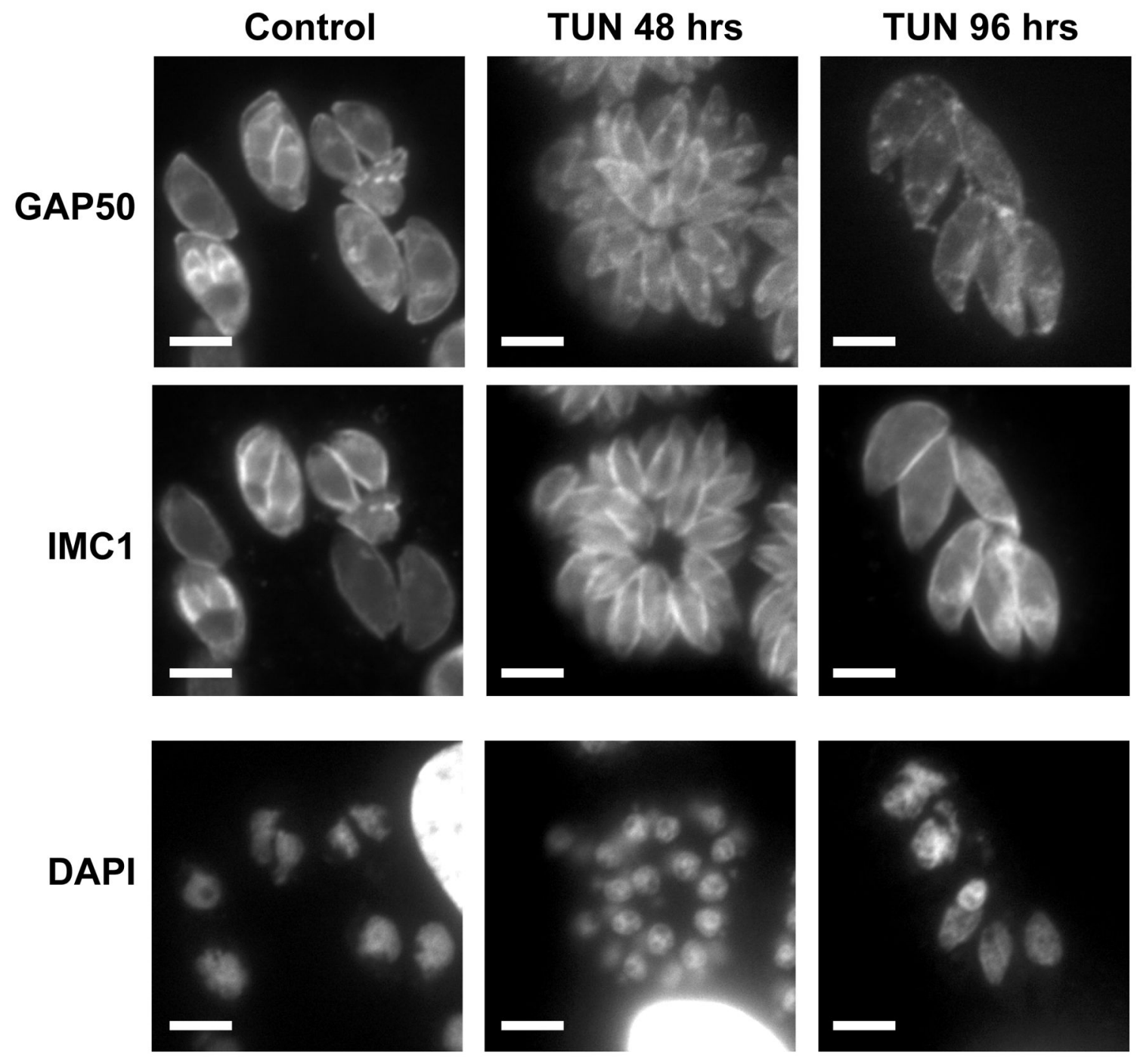

Figure 4.

GAP50 expression and nuclear morphology are affected by long-term treatment with tunicamycin.

Toxoplasma tachyzoites expressing a GAP50-YFP fusion protein were treated for 48 or 96 hours in the presence or absence of $5 \mu \mathrm{gr} / \mathrm{ml}$ tunicamycin. The membrane skeletal protein IMC1 was visualized by indirect immunofluorescence and the nuclear and apicoplast DNA by staining with DAPI. The exposure time for GAP50-YFP was $200 \mathrm{msec}$ for the control and 48 hour incubation, and 2 seconds for the 96 hour incubation. Scale bars: $5 \mu \mathrm{m}$. 

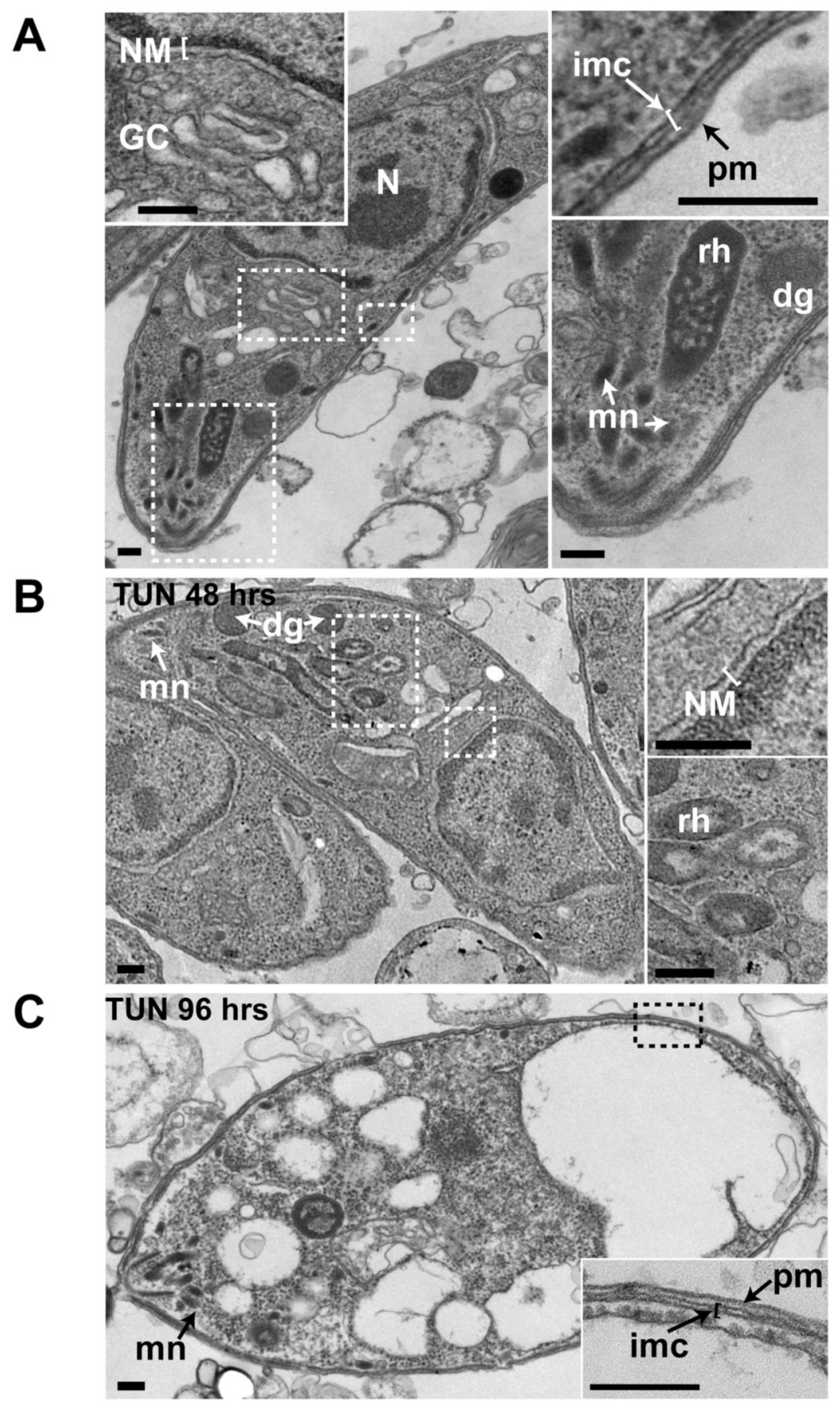

Figure 5.

Inhibition of N-glycosylation and Toxoplasma ultrastructure.

Toxoplasma tachyzoites were incubated in the presence of DMSO (A) or in the presence of (in the text you stated that you used $10 \mathrm{ug} / \mathrm{mL}$; not sure which one is which) $5 \mu \mathrm{g} / \mathrm{ml}$ tunicamycin for (B) 48 hours or (C) 96 hours. Scale bars: $200 \mathrm{~nm}$. Insets show details of the parasite Golgi complex (GC), nuclear membrane (NM), inner membrane complex (imc), plasma membrane $(\mathrm{PM})$, dense granules (dg), rhoptries (rh), and micronemes (mn). 


\section{Pulse 60' Chase}

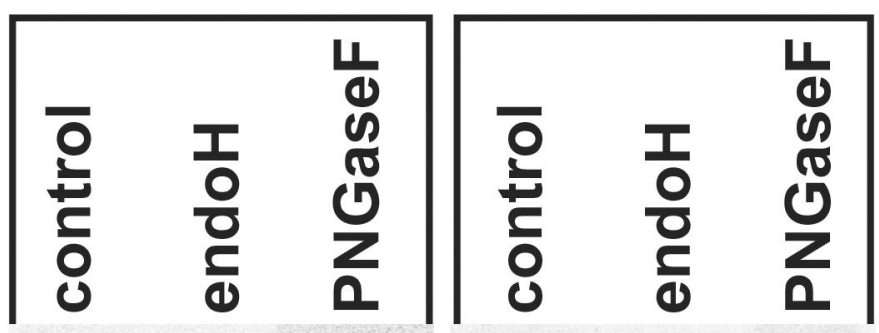

\section{$50 \mathrm{kDa}-$}
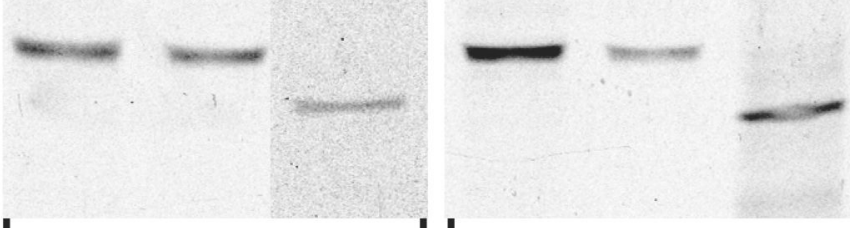

$50 \mathrm{kDa}-$

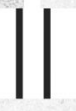

\section{- GAP50 + OS GAP50 - OS}

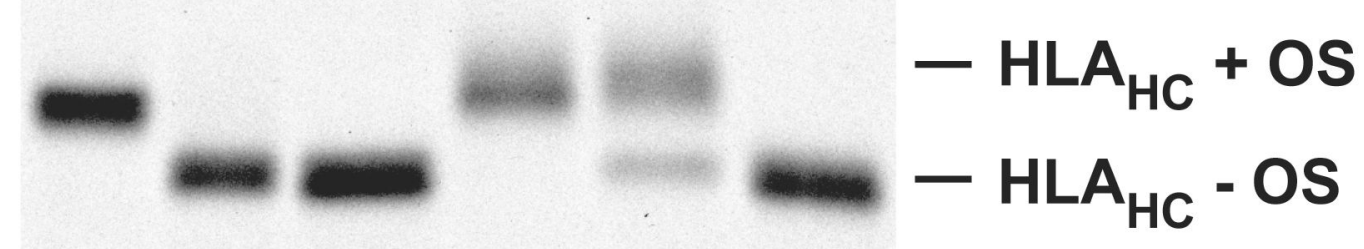

Figure 6.

Toxoplasma N-linked oligosaccharides are never sensitive to endoglycosidase $\mathrm{H}$. Uninfected or Toxoplasma-infected HFF cells were pulse labeled with $\left[{ }^{35} \mathrm{~S}\right]$ methionine/ cysteine for 10 minutes and subsequently chased for 60 minutes in the presence of excess unlabeled methionine and cysteine. GAP50 and the HLA class I heavy chains were immunoprecipitated from the Toxoplasma-infected or uninfected HFF cells, respectively, and subjected to digestion with endoglycosidase $\mathrm{H}$ or PNGase $\mathrm{F}$. The positions of the fully glycosylated (GAP50+OS, HLA $\mathrm{HC}^{+O S}$ ) and deglycosylated proteins (GAP50-OS, $\mathrm{HLA}_{\mathrm{HC}^{-}}$ OS) are indicated. 


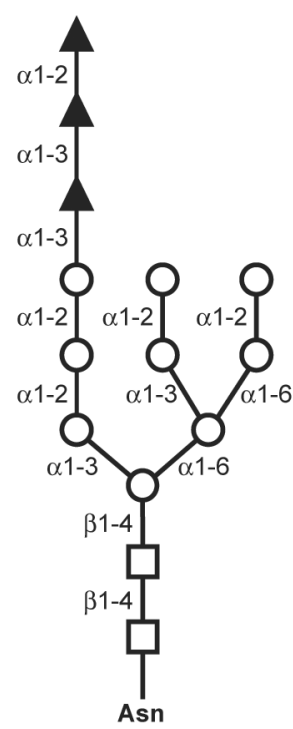

A

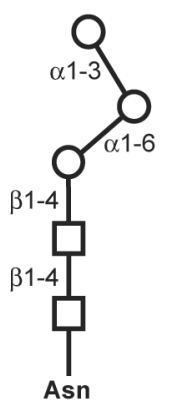

B

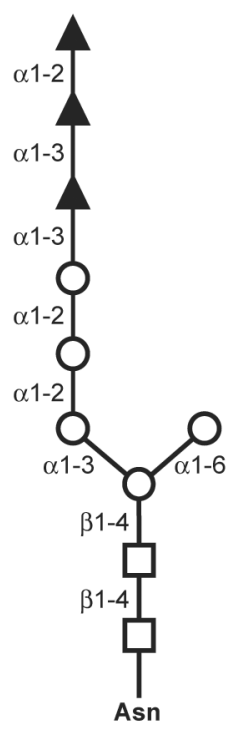

C

Figure 7.

A) Structure of the typical core N-linked oligosaccharide on newly synthesized animal and yeast glycoproteins.

B) Minimal N-linked oligosaccharide structure required for sensitivity to endoglycosidase $\mathrm{H}$ [21].

C) Predicted structure of N-linked oligosaccharide on newly synthesized glycoproteins in Toxoplasma gondii. 